\title{
Increase in participation but decrease in performance in age group mountain marathoners in the 'Jungfrau Marathon': a Swiss phenomenon?
}

\author{
Beat Knechtle ${ }^{1,2^{*}}$, Thomas Rosemann ${ }^{2}$, Matthias A. Zingg ${ }^{2}$ and Christoph A. Rüst ${ }^{2}$
}

\begin{abstract}
Participation and performance trends for age group marathoners have been investigated for large city marathons such as the 'New York City Marathon' but not for mountain marathons. This study investigated participation and trends in performance and sex difference in the mountain marathon 'Jungfrau Marathon' held in Switzerland from 2000 to 2014 using single and mixed effects regression analyses. Results were compared to a city marathon (Lausanne Marathon) also held in Switzerland during the same period. Sex difference was calculated using the equation ([race time in women] - [race time in men]/[race time in men] $\times 100$ ). Changes in sex differences across calendar years and were investigated using linear regression models. In 'Jungfrau Marathon', participation in all female and male age groups increased with exception of women in age groups 18-24 and men in age groups 30-34, 40-44 and 60-64 years where participation remained unchanged. In 'Lausanne Marathon', participation increased in women in age groups 30-34 to 40-44 years. In men, participation increased in age groups 25-29 to 44-44 years and 50-54 years. In'Jungfrau Marathon' runners became slower across years in age groups 18-24 to 70-74 years. In 'Lausanne Marathon', runners became slower across years in age groups 18-24 and 30-34 to 65-69 years, but not for 25-29, 70-74 and 75-79 years. In 'Jungfrau Marathon', sex difference increased in age groups 25-29 (from 4 to 10 \%) and 60-64 years (from 3 to $8 \%$ ) but decreased in age group 40-44 years (from 12 to $6 \%$ ). In 'Lausanne Marathon', the sex difference showed no changes. In summary, participation increased in most female and male age groups but performance decreased in most age groups for both the mountain marathon 'Jungfrau Marathon' and the city marathon 'Lausanne Marathon.'The sex differences were lower in the 'Jungfrau Marathon' ( 6-7 \%) compared to the 'Lausanne Marathon' where the sex difference was 10-12\% from age groups 18-24 to 55-59 years. These unexpected findings might be a typical Swiss phenomenon. Future studies need to investigate whether this trend can also be found in other endurance sports events held in Switzerland and other mountain marathons held in other countries.
\end{abstract}

Keywords: Female, Male, Runner, Master

\section{Background}

Marathon running is of high popularity where marathon races are mainly held as city marathons in large cities (Jokl et al. 2004; Lepers and Cattagni 2012; Leyk et al. 2007, 2009, 2010). Participation and performance

\footnotetext{
*Correspondence: beat.knechtle@hispeed.ch

${ }^{1}$ Gesundheitszentrum St. Gallen, Vadianstrasse 26, 9001 St. Gallen,

Switzerland

Full list of author information is available at the end of the article
}

trends in elite and age group city marathoners are well investigated (Ahmadyar et al. 2015; Anthony et al. 2014; Aschmann et al. 2013; Cribari et al. 2013, Jokl et al. 2004, Lepers and Cattagni 2012; Leyk et al. 2007, 2009), but very little is known for mountain marathoners (Zingg et al. 2013).

In a large city marathon such as the 'New York City Marathon', most of the successful finishers are age group or master runners (Jokl et al. 2004), defined as athletes older than 35 years (Reaburn and Dascombe 2008). In

\section{贷 Springer}


the 'New York City Marathon', participation increased in master age groups between 1983 and 1999 at a higher rate compared to other age groups and master runners improved their race times at a greater rate compared to younger athletes (Jokl et al. 2004). Lepers and Cattagni (2012) investigated for the 'New York City Marathon' participation and performance trends in master runners during a longer period from 1980 to 2009. During this period, the participation of male master runners increased to a greater extent compared to female master runners. Male master runners older than 64 years and female master runners older than 44 years improved their performance (Lepers and Cattagni 2012).

Apart from marathon running in a city marathon, athletes can also compete in mountain marathons (http://www.mountainrunning.com, http://www. wmra.info). However, in contrast to city marathons (Jokl et al. 2004, Lepers and Cattagni 2012, Leyk et al. $2007,2009,2010$ ) we have no data on the participation and performance trends in age group mountain marathoners. Since mountain running is of increasing popularity (http://www.mountainrunning.com, http://www.wmra.info), the aim of this study was to investigate participation and performance trends in age group marathoners competing in the 'Jungfrau Marathon' held in the Swiss Alps. This marathon race started in 1993 and is actually the most famous mountain marathon in Europe. In 2007 and 2012, the 'Jungfrau Marathon' was held as the World Championship in mountain running. Based upon the findings for a large city marathon such as the 'New York City Marathon', we hypothesized that age group mountain marathoners competing in the 'Jungfrau Marathon' would increase their participation and improve their performance. To compare findings for this mountain marathon, we analyzed also race data from a city marathon (Lausanne Marathon) held during the same time period and in the same country.

\section{Methods}

\section{Ethics}

All procedures used in the study were approved by the Institutional Review Board of Kanton St. Gallen, Switzerland with a waiver of the requirement for informed consent of the participants given the fact that the study involved the analysis of publicly available data.

\section{The races}

In this study, all athletes who finished the 'Jungfrau Marathon' and the 'Lausanne Marathon' between 2000 and 2014 were analysed for participation, running time, age, and sex.The data set for this study was obtained from the race website http://www.jungfrau-marathon.ch for the 'Jungfrau Marathon' and from http://de.lausanne-marathon.com for the 'Lausanne Marathon'.

The 'Jungfrau Marathon' in Switzerland started in 1993, after mountain running was becoming increasingly popular in Europe. Nowadays, the 'Jungfrau Marathon' is one of the most popular mountain marathons in the world. The race is held annually in autumn. The 'Jungfrau Marathon' starts in Interlaken (565 m above sea level) and finishes at the 'Kleine Scheidegg' (2095 m above sea level). The course covers $1830 \mathrm{~m}$ of altitude gain and $305 \mathrm{~m}$ of loss in altitude. The first quarter of the race is mostly flat and only $\sim 300 \mathrm{~m}$ of altitude difference are covered up to the first half of the marathon. The end of the race stands at $2095 \mathrm{~m}$ above sea level, right next to the world famous Eiger. In 2007 and 2012, the 'Jungfrau Marathon' was the official World Championship in mountain running, which is held annually along a different race each year.

The 'Lausanne Marathon' started in 1992 and is the second most important city marathon in Switzerland behind the 'Zürich Marathon. The race is held annually in autumn in the city of Lausanne, on the border of Lake Léman, on a flat course. After the first half, the course turns and goes back to Lausanne. Overall, the race has minus 36 meters of altitude change. Apart from the marathon, the 'Lausanne Marathon' hosts the annual semimarathon Swiss Championship, a quarter marathon race, and a mini race for kids. All these races make the 'Lausanne Marathon' one of the most important marathon events held in Switzerland.

\section{Statistical analysis}

All athletes in all age groups competing in both the 'Jungfrau Marathon' and the 'Lausanne Marathon' were considered for analysis by 5-year age groups from 18-25 to 75-79 years. Trends in participation and in the men-towomen ratio across calendar years and across age groups were analyzed using single linear regression analyses. To analyze changes in performance, a mixed-effects regression model with finisher as random variable to consider finishers who completed several races was used. We included sex and calendar year as fixed variables. Absolute sex difference was calculated using the equation ([absolute race time in women] - [absolute race time in men]/[absolute race time in men] $\times 100$ ). Changes in sex differences across calendar years and were investigated using linear regression models. Statistical analyses were performed using IBM SPSS Statistics (Version 22, IBM SPSS, Chicago, IL, USA). Significance was accepted at $\mathrm{P}<0.05$ (two-sided for $t$ tests). Data in the text and tables are given as mean \pm standard deviation (SD). 


\section{Results}

\section{Participation trends}

In the 'Jungfrau Marathon', participation in all female and male age groups increased with exception of women in age group 18-24 years and men in age groups 30-34, 40-44 and 55-59 years where participation remained unchanged (Table 1). In the 'Lausanne Marathon', participation increased in women only in age groups 30-34 to 40-44 years. In men, participation increased in age groups 25-29 to 44-44 years and 60-64 years (Table 1).

Table 2 summarizes the trends in the men-to-women ratio across age groups and across calendar years for both the 'Jungfrau Marathon' and the 'Lausanne Marathon'. In the 'Jungfrau Marathon', the men-to-women ratio increased across age groups in 2008, 2010, and 2012-2014. Across calendar years, the ratio decreased in age groups $30-34$ to $50-54$ and $60-64$ to $65-69$ years. For the 'Lausanne Marathon', the men-to-women ratio increased across age groups in 2000, 2002, 2003, 2005, 2006 and 2008. Across calendar years, the ratio decreased in age group 55-59 years and remained unchanged for all other age groups.

\section{Performance trends}

Table 3 presents the race times in the 'Jungfrau Marathon' and the 'Lausanne Marathon' for women and men by age group. In the 'Jungfrau Marathon', race time showed a significant and positive change for sex for all age groups (i.e. the runners became slower across age groups) with the exception of age group 75-79 years (Table 4). For calendar year, the changes were significant and positive (i.e. the runners became slower across years) for age groups 18-24 to 70-74 years. In the 'Lausanne Marathon', race time showed a significant and positive change (i.e. the runners became slower across age groups) for sex for all age groups from 18-24 to 70-74 years (Table 5). For calendar year, the changes were significant and positive (i.e. the runners became slower across years) for age groups 18-24 and 30-34 to 65-69 years, but not for 25-29, $70-74$ and $75-79$ years.

\section{Sex difference}

In the 'Jungfrau Marathon', the sex difference increased in age groups 25-29 (from 4 to $10 \%$ ) and 60-64 years (from 3 to $8 \%$ ) but decreased in age group 40-44 years (from 12 to $6 \%$ ) (Table 6). For age groups $18-24,30-34,35-39$, $45-49,50-54,55-59,60-64,65-69$, and 70-74 years, the values were $7.3 \pm 2.9,6.3 \pm 2.0,6.2 \pm 1.3,7.5 \pm 1.3$, $7.5 \pm 2.1,6.4 \pm 2.2,5.2 \pm 2.9$, and $6.0 \pm 4.5 \%$, respectively. In the 'Lausanne Marathon', however, the sex difference showed no changes. The values for the sex difference were $10.4 \pm 8.1,10.6 \pm 5.5,11.2 \pm 4.5$, $9.1 \pm 3.5$, $10.2 \pm 2.7,12.1 \pm 5.7,10.7 \pm 5.8,11.7 \pm 7.2,16.0 \pm 8.1$,
$28.3 \pm 9.7$, and $27.5 \pm 35.1 \%$ for age groups $18-24$, $25-29,30-34,35-39,40-44,45-49,50-54,55-59$, 60-64, and 65-69, respectively (Table 6).

\section{Discussion}

The aim of the present study was investigate participation and performance trends in the mountain marathon 'Jungfrau Marathon' with the hypothesis that participation would increase and performance would improve as it has been reported for a large city marathon such as the 'New York City Marathon'. The main findings were for both the 'Jungfrau Marathon' and the 'Lausanne Marathon' that (1) participation increased in most age groups but (2) performance decreased in most age groups for both women and men.

\section{Participation trends}

City marathons have been held for decades whereas the first official marathon was in the 1896 Summer Olympics (http://www.olympic.org/athens-1896-summerolympics). Mountain marathon became popular much later (http://www.wmra.info/). The present findings in participation and performance in these mountain marathoners competing in the 'Jungfrau Marathon' might be specific for Switzerland. Teutsch et al. (2013) investigated participation and performance trends in age group inline skaters competing between 1998 and 2009 in the longest inline race in Europe, the 'Inline One-Eleven' over $111 \mathrm{~km}$, which was also held in Switzerland. During the 12-year period, overall participation increased until 2003 but decreased thereafter. The relative participation in athletes younger than 40 years old decreased while relative participation increased for athletes older than 40 years. The race times of the best female and male skaters stabilized across years (Teutsch et al. 2013). In the 120-km ultra-endurance mountain bike race the 'Swiss Bike Masters' held in Switzerland from 1995 to 2009, similar findings were reported (Gloor et al. 2013; Haupt et al. 2013). The number of male finishers decreased while women's participation has remained low. Performances of the annual fastest women improved, while performances of the annual fastest men remained unchanged (Gloor et al. 2013). However, this trend of an increase in participation with impairment in performance was not obvious in all races held in Switzerland. In 'Ironman Switzerland' held between 1995 and 2011, the number of finishers increased the successful finishers were able to improve their race times (Rüst et al. 2012). In the 'Powerman Zofingen' long-distance duathlon (10-km run, $150-\mathrm{km}$ cycle, and 30-km run) held from 2002 to 2011 in Switzerland, the participation remained across years fairly stable and similarly the performance in running and cycling times were also fairly for both male and female 
Table 1 Participation in women and men in age groups in the mountain (upper two panels) and the city marathon (lower two panels)

\begin{tabular}{|c|c|c|c|c|c|c|c|c|c|c|c|c|c|c|c|c|c|c|}
\hline Sex & Age group & 2000 & 2001 & 2002 & 2003 & 2004 & 2005 & 2006 & 2007 & 2008 & 2009 & 2010 & 2011 & 2012 & 2013 & 2014 & $r^{2}$ & $\mathrm{p}$ \\
\hline \multicolumn{19}{|c|}{ Jungfrau Marathon } \\
\hline \multirow[t]{11}{*}{ Women } & $18-24$ & 4 & 9 & 22 & 12 & 15 & 11 & 11 & 12 & 10 & 8 & 12 & 14 & 27 & 23 & 13 & 0.18 & 0.109 \\
\hline & $25-29$ & 28 & 19 & 39 & 45 & 41 & 47 & 37 & 48 & 37 & 44 & 46 & 46 & 86 & 62 & 60 & 0.56 & 0.0012 \\
\hline & $30-34$ & 62 & 62 & 78 & 67 & 75 & 71 & 82 & 92 & 69 & 58 & 66 & 97 & 141 & 97 & 96 & 0.37 & 0.015 \\
\hline & $35-39$ & 70 & 85 & 109 & 97 & 130 & 114 & 103 & 120 & 114 & 106 & 91 & 94 & 168 & 129 & 116 & 0.27 & 0.046 \\
\hline & $40-44$ & 95 & 87 & 137 & 112 & 122 & 167 & 162 & 199 & 171 & 198 & 173 & 157 & 279 & 172 & 170 & 0.52 & 0.002 \\
\hline & $45-49$ & 63 & 66 & 125 & 91 & 109 & 120 & 135 & 162 & 153 & 177 & 168 & 174 & 326 & 178 & 187 & 0.64 & 0.0003 \\
\hline & $50-54$ & 47 & 43 & 42 & 43 & 48 & 64 & 67 & 80 & 76 & 102 & 110 & 104 & 231 & 117 & 121 & 0.61 & 0.005 \\
\hline & $55-59$ & 25 & 25 & 11 & 32 & 25 & 34 & 41 & 34 & 29 & 61 & 35 & 52 & 123 & 57 & 52 & 0.44 & 0.006 \\
\hline & $60-64$ & 10 & 12 & 7 & 12 & 11 & 16 & 13 & 20 & 19 & 19 & 22 & 22 & 43 & 22 & 29 & 0.66 & 0.0002 \\
\hline & $65-69$ & 1 & 3 & & 5 & 2 & 2 & 3 & 5 & 5 & 5 & 5 & 3 & 18 & 6 & 8 & 0.39 & 0.012 \\
\hline & $70-74$ & 2 & 1 & & & & 1 & & & 1 & & 1 & 2 & 6 & 4 & 5 & 0.41 & 0.0096 \\
\hline \multirow{11}{*}{ Men } & $25-29$ & 120 & 127 & 79 & 123 & 136 & 114 & 123 & 143 & 135 & 128 & 128 & 128 & 230 & 156 & 190 & 0.45 & 0.0061 \\
\hline & $30-34$ & 336 & 330 & 163 & 279 & 335 & 272 & 288 & 317 & 257 & 252 & 245 & 285 & 417 & 268 & 286 & 0.004 & 0.816 \\
\hline & $35-39$ & 513 & 524 & 313 & 526 & 540 & 496 & 463 & 458 & 430 & 418 & 380 & 365 & 513 & 348 & 324 & 0.29 & 0.035 \\
\hline & $40-44$ & 525 & 535 & 383 & 562 & 623 & 670 & 665 & 739 & 680 & 697 & 617 & 586 & 877 & 504 & 445 & 0.05 & 0.384 \\
\hline & $45-49$ & 445 & 445 & 361 & 485 & 539 & 550 & 576 & 637 & 645 & 645 & 648 & 687 & 993 & 602 & 608 & 0.53 & 0.0019 \\
\hline & $50-54$ & 335 & 322 & 452 & 346 & 393 & 409 & 437 & 450 & 444 & 509 & 493 & 445 & 894 & 504 & 530 & 0.43 & 0.0074 \\
\hline & $55-59$ & 182 & 203 & 251 & 205 & 194 & 238 & 223 & 274 & 255 & 281 & 282 & 294 & 463 & 288 & 289 & 0.52 & 0.0023 \\
\hline & $60-64$ & 134 & 117 & 148 & 112 & 108 & 106 & 97 & 133 & 129 & 135 & 126 & 139 & 245 & 135 & 150 & 0.19 & 0.096 \\
\hline & $65-69$ & 36 & 36 & 50 & 34 & 45 & 53 & 54 & 51 & 39 & 56 & 59 & 49 & 111 & 60 & 48 & 0.26 & 0.035 \\
\hline & $70-74$ & 8 & 4 & 6 & 3 & 7 & 8 & 5 & 8 & 11 & 14 & 11 & 10 & 27 & 18 & 23 & 0.65 & 0.0003 \\
\hline & $75-79$ & & & 1 & 1 & 1 & 1 & 1 & 2 & 1 & 1 & 1 & 1 & 3 & 2 & 4 & 0.58 & 0.0009 \\
\hline \multicolumn{19}{|c|}{ Lausanne Marathon } \\
\hline \multirow[t]{10}{*}{ Women } & $18-24$ & 11 & 24 & 8 & 13 & 13 & 17 & 15 & 6 & 6 & 4 & 10 & 7 & 10 & 17 & 15 & 0.04 & 0.44 \\
\hline & $35-39$ & 56 & 80 & 51 & 54 & 50 & 60 & 47 & 28 & 31 & 33 & 25 & 32 & 35 & 36 & 25 & 0.65 & 0.0002 \\
\hline & $40-44$ & 58 & 61 & 65 & 55 & 65 & 64 & 77 & 44 & 32 & 42 & 34 & 46 & 42 & 33 & 38 & 0.52 & 0.0021 \\
\hline & $45-49$ & 26 & 55 & 41 & 45 & 35 & 67 & 47 & 33 & 29 & 39 & 35 & 62 & 29 & 38 & 28 & 0.03 & 0.48 \\
\hline & $50-54$ & 24 & 34 & 16 & 32 & 25 & 22 & 30 & 25 & 16 & 17 & 27 & 21 & 22 & 36 & 17 & 0.02 & 0.54 \\
\hline & $55-59$ & 6 & 13 & 9 & 13 & 19 & 14 & 11 & 17 & 13 & 10 & 12 & 16 & 15 & 13 & 15 & 0.14 & 0.16 \\
\hline & $60-64$ & 4 & 6 & 0 & 2 & 8 & & 4 & 17 & 4 & 5 & 8 & 8 & 4 & 8 & 6 & 0.09 & 0.26 \\
\hline & $65-69$ & 1 & 3 & 1 & 1 & 2 & & & 8 & 2 & 1 & 2 & & 1 & 3 & 3 & 0.01 & 0.69 \\
\hline & $70-74$ & & & & & 1 & & & 1 & & & & & 1 & & & 0.005 & 0.78 \\
\hline & $75-79$ & & 1 & & & & & & & & & & & & 1 & & 1.01 & 1.0 \\
\hline \multirow[t]{12}{*}{ Men } & $18-24$ & 50 & 55 & 43 & 36 & 28 & 34 & 34 & 33 & 27 & 41 & 42 & 36 & 57 & 56 & 43 & 0.01 & 0.66 \\
\hline & $25-29$ & 140 & 155 & 97 & 114 & 111 & 84 & 95 & 84 & 74 & 85 & 93 & 71 & 82 & 109 & 93 & 0.39 & 0.012 \\
\hline & $30-34$ & 225 & 259 & 225 & 198 & 217 & 155 & 178 & 139 & 112 & 153 & 122 & 120 & 129 & 139 & 110 & 0.78 & $<0.0001$ \\
\hline & $35-39$ & 311 & 318 & 314 & 285 & 284 & 263 & 209 & 210 & 158 & 192 & 209 & 192 & 141 & 173 & 140 & 0.87 & $<0.0001$ \\
\hline & $40-44$ & 278 & 305 & 275 & 294 & 325 & 336 & 295 & 267 & 213 & 225 & 223 & 214 & 217 & 222 & 182 & 0.65 & $<0.0001$ \\
\hline & $45-49$ & 209 & 188 & 186 & 197 & 233 & 263 & 227 & 183 & 164 & 159 & 214 & 186 & 172 & 188 & 153 & 0.19 & 0.097 \\
\hline & $50-54$ & 138 & 167 & 150 & 156 & 148 & 170 & 148 & 126 & 116 & 112 & 139 & 103 & 109 & 118 & 125 & 0.53 & 0.0019 \\
\hline & $55-59$ & 73 & 71 & 74 & 76 & 91 & 97 & 74 & 87 & 67 & 57 & 66 & 65 & 59 & 62 & 68 & 0.26 & 0.050 \\
\hline & $60-64$ & 40 & 39 & 48 & 19 & 41 & 37 & 41 & 38 & 30 & 34 & 38 & 49 & 34 & 41 & 33 & 0.0008 & 0.91 \\
\hline & $65-69$ & 15 & 14 & 15 & 8 & 11 & 12 & 14 & 17 & 12 & 12 & 11 & 13 & 7 & 18 & 13 & 0.004 & 0.81 \\
\hline & $70-74$ & 3 & 4 & 2 & 3 & 4 & 6 & 2 & 5 & 4 & 1 & 5 & 7 & 4 & 3 & 5 & 0.08 & 0.29 \\
\hline & $75-79$ & & 1 & & & 1 & 1 & 1 & 1 & 2 & 1 & 1 & 1 & & 2 & & 0.06 & 0.34 \\
\hline
\end{tabular}


Table 2 The men-to-women ratio for the mountain and the city marathon across age groups and calendar years

\begin{tabular}{|c|c|c|c|c|c|c|c|c|c|c|c|c|c|c|c|c|c|}
\hline Age group & 2000 & 2001 & 2002 & 2003 & 2004 & 2005 & 2006 & 2007 & 2008 & 2009 & 2010 & 2011 & 2012 & 2013 & 2014 & $r^{2}$ & $p$ \\
\hline \multicolumn{18}{|c|}{ Jungfrau Marathon } \\
\hline $18-24$ & 8.2 & 4.6 & 0.6 & 3.5 & 4.3 & 6.5 & 5.8 & 6.2 & 4.8 & 6.7 & 4.6 & 4.2 & 3.4 & 2.9 & 4.3 & 0.03 & 0.52 \\
\hline $25-29$ & 4.2 & 6.6 & 2.0 & 2.7 & 3.3 & 2.4 & 3.3 & 2.9 & 3.6 & 2.9 & 2.7 & 2.7 & 2.6 & 2.5 & 3.1 & 0.19 & 0.10 \\
\hline $30-34$ & 5.4 & 5.3 & 2.0 & 4.1 & 4.4 & 3.8 & 3.5 & 3.4 & 3.7 & 4.3 & 3.7 & 2.9 & 2.9 & 2.7 & 2.9 & 0.34 & 0.02 \\
\hline $35-39$ & 7.3 & 6.1 & 2.8 & 5.4 & 4.1 & 4.3 & 4.4 & 3.8 & 3.7 & 3.9 & 4.1 & 3.8 & 3.0 & 2.6 & 2.7 & 0.54 & 0.001 \\
\hline $40-44$ & 5.5 & 6.1 & 2.7 & 5.0 & 5.1 & 4.0 & 4.1 & 3.7 & 3.9 & 3.5 & 3.5 & 3.7 & 3.1 & 2.9 & 2.6 & 0.57 & 0.001 \\
\hline $45-49$ & 7.0 & 6.7 & 2.8 & 5.3 & 4.9 & 4.5 & 4.2 & 3.9 & 4.2 & 3.6 & 3.8 & 3.9 & 3.0 & 3.3 & 3.2 & 0.53 & 0.002 \\
\hline $50-54$ & 7.1 & 7.4 & 10.7 & 8.0 & 8.1 & 6.3 & 6.5 & 5.6 & 5.8 & 4.9 & 4.4 & 4.2 & 3.8 & 4.3 & 4.3 & 0.73 & $<0.0001$ \\
\hline $55-59$ & 7.2 & 8.1 & 22.8 & 6.4 & 7.7 & 7.0 & 5.4 & 8.0 & 8.7 & 4.6 & 8.0 & 5.6 & 3.7 & 5.0 & 5.5 & 0.21 & 0.88 \\
\hline $60-64$ & 13.4 & 9.7 & 21.1 & 9.3 & 9.8 & 6.6 & 7.4 & 6.6 & 6.7 & 7.1 & 5.7 & 6.3 & 5.6 & 6.1 & 5.1 & 0.49 & 0.003 \\
\hline $65-69$ & 36.0 & 12.0 & & 6.8 & 22.5 & 26.5 & 18.0 & 10.2 & 7.8 & 11.2 & 11.8 & 16.3 & 6.1 & 10.0 & 6.0 & 0.35 & 0.02 \\
\hline $70-74$ & 4.0 & 4.0 & & & & 8.0 & & & 11.0 & & 11.0 & 5.0 & 4.5 & 4.5 & 4.6 & 0.00 & 0.85 \\
\hline$r^{2}$ & 0.19 & 0.24 & 0.12 & 0.05 & 0.18 & 0.30 & 0.09 & 0.04 & 0.71 & 0.01 & 0.64 & 0.33 & 0.61 & 0.54 & 0.46 & & \\
\hline$p$ & 0.18 & 0.13 & 0.30 & 0.49 & 0.19 & 0.08 & 0.36 & 0.56 & 0.001 & 0.73 & 0.002 & 0.06 & 0.004 & 0.01 & 0.02 & & \\
\hline \multicolumn{18}{|c|}{ Lausanne Marathon } \\
\hline $18-24$ & 4.5 & 2.2 & 5.3 & 2.7 & 2.1 & 2.0 & 2.2 & 5.5 & 4.5 & 10.2 & 4.2 & 5.1 & 5.7 & 3.2 & 2.8 & 0.05 & 0.41 \\
\hline $25-29$ & 7.0 & 2.6 & 2.9 & 4.9 & 4.4 & 1.9 & 3.5 & 4.6 & 3.7 & 4.0 & 6.6 & 3.7 & 2.8 & 5.7 & 4.8 & 0.01 & 0.71 \\
\hline $30-34$ & 6.6 & 4.3 & 5.0 & 5.2 & 7.2 & 3.4 & 4.4 & 4.3 & 5.6 & 4.9 & 9.3 & 5.4 & 3.5 & 4.7 & 3.3 & 0.02 & 0.57 \\
\hline $35-39$ & 5.5 & 3.9 & 6.1 & 5.2 & 5.6 & 4.3 & 4.4 & 7.5 & 5.0 & 5.8 & 8.3 & 6.0 & 4.0 & 4.8 & 5.6 & 0.01 & 0.66 \\
\hline $40-44$ & 4.7 & 5.0 & 4.2 & 5.3 & 5.0 & 5.2 & 3.8 & 6.0 & 6.6 & 5.3 & 6.5 & 4.6 & 5.1 & 6.7 & 4.7 & 0.14 & 0.17 \\
\hline $45-49$ & 8.0 & 3.4 & 4.5 & 4.3 & 6.6 & 3.9 & 4.8 & 5.5 & 5.6 & 4.0 & 6.1 & 3.0 & 5.9 & 4.9 & 5.4 & 0.01 & 0.73 \\
\hline $50-54$ & 5.7 & 4.9 & 9.3 & 4.8 & 5.9 & 7.7 & 4.9 & 5.0 & 7.2 & 6.5 & 5.1 & 4.9 & 4.9 & 3.2 & 7.3 & 0.06 & 0.39 \\
\hline $55-59$ & 12.1 & 5.4 & 8.2 & 5.8 & 4.7 & 6.9 & 6.7 & 5.1 & 5.1 & 5.7 & 5.5 & 4.0 & 3.9 & 4.7 & 4.5 & 0.46 & 0.005 \\
\hline $60-64$ & 10.0 & 6.5 & & 9.5 & 5.1 & & 10.2 & 2.2 & 7.5 & 6.8 & 4.7 & 6.1 & 8.5 & 5.1 & 5.5 & 0.14 & 0.20 \\
\hline $65-69$ & 15.0 & 4.6 & 15.0 & 8.0 & 5.5 & & & 2.1 & 6.0 & 12.0 & 5.5 & & 7.0 & 6.0 & 4.3 & 0.20 & 0.13 \\
\hline $70-74$ & & & & & 4.0 & & & 5.0 & & & & & 4.0 & & & 0.02 & 0.90 \\
\hline $75-79$ & & 1.0 & & & & & & & & & & & & 2.0 & & & \\
\hline$r^{2}$ & 0.61 & 0.02 & 0.65 & 0.60 & 0.02 & 0.83 & 0.73 & 0.23 & 0.45 & 0.08 & 0.12 & 0.00 & 0.17 & 0.04 & 0.19 & & \\
\hline$p$ & 0.007 & 0.64 & 0.008 & 0.008 & 0.66 & 0.001 & 0.003 & 0.13 & 0.03 & 0.42 & 0.31 & 0.91 & 0.20 & 0.53 & 0.20 & & \\
\hline
\end{tabular}

elite duathletes (Rüst et al. 2013). An increase in participation and a decrease in performance are most probably not specific for races held in Switzerland, although not all mentioned studies investigated the trends for age group athletes. The fact that the participation in the mountain marathon grew but not in the city marathon might be due to the fact that running on concrete surface is associated with increased potential of injury (Van der Worp et al. 2015).

\section{Performance trends}

The finding that age group athletes became slower in the mountain marathon 'Jungfrau Marathon' is controversial to reported findings for a large city marathon such as the 'New York City Marathon' (Jokl et al. 2004; Lepers and Cattagni 2012). The most likely explanation for this difference in the fact that we included all recorded finishers for each age group for our analysis while Jokl et al. (2004) considered the fastest 50 women and men for each age group and Lepers and Cattagni (2012) the ten fastest women and men. With an increasing participation in the 'New York City Marathon' the density of elite runners might become higher and therefore the fastest 10 and fastest 50 finishers became faster. On the other side, an increase of the participation might lower the average performance level. The selection of a fixed number of athletes for each age group (i.e. the ten fastest) may lead to a selection bias. When we consider all successful finishers for each age group, the number of athletes for each age group will increase and also include slow and weak runners who finish just within the time limit. Therefore, the mean marathon race time in an age group with a large number of finishers will be relatively high.

A further interesting finding was that also the race times in the city marathon 'Lausanne Marathon' became slower. Similarly to the 'Jungfrau Marathon', all finishers were included in each age group. The decrease in performance in both the 'Jungfrau Marathon' and the 


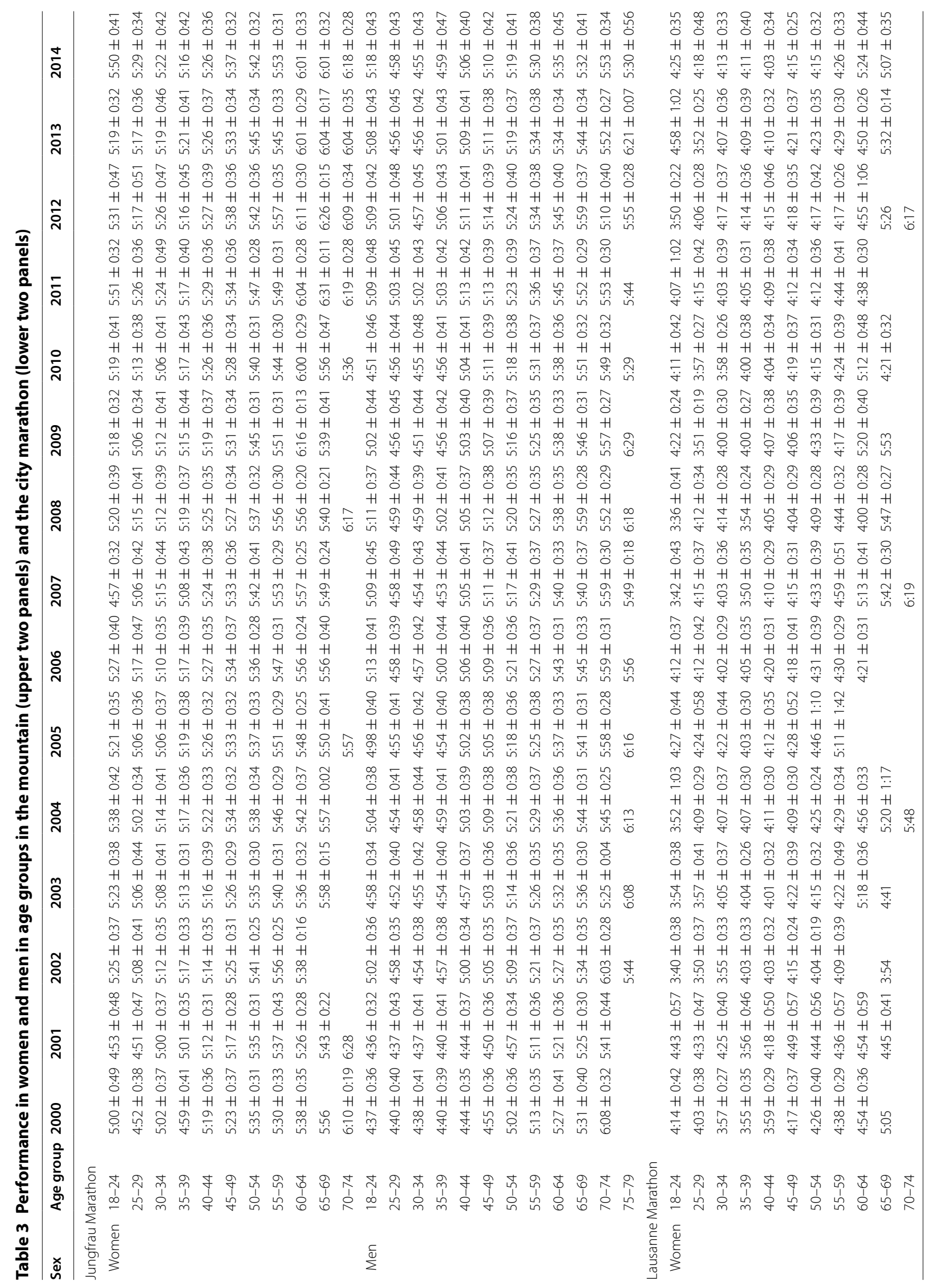


$\stackrel{m}{m} m \infty$ H H H H H H H H H H H

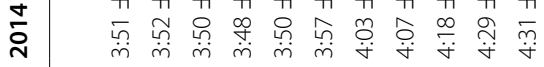

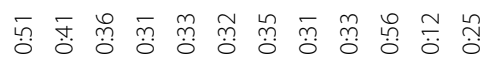
H H H H H H H H H H H H

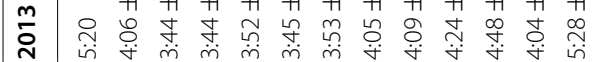

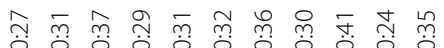
H H H H H H H H H H H

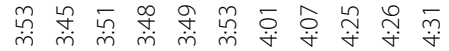

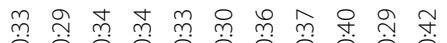
H H H H H H H H H H H $\bar{i}$ in m $\mathrm{H}+\mathrm{H} \quad \mathrm{H}$ H $\mathrm{H}$ H $\mathrm{H}$ H H H H H

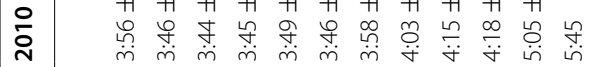

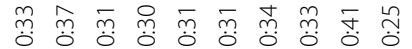

H H H H H H H H H H

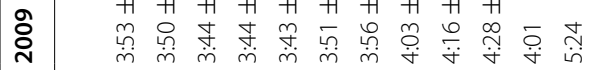

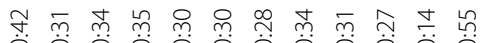
H H H H H H H H H H H H

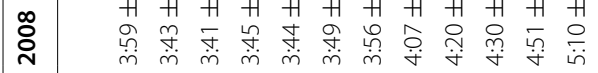

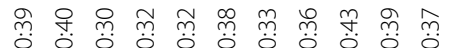
H H H H H H H H H H H

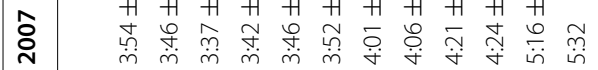
m

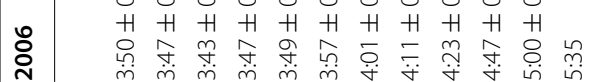

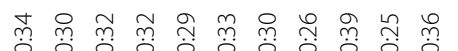

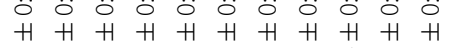

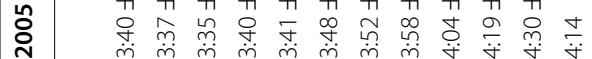

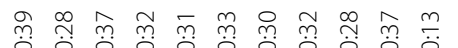
$20 \hat{O}$
$H$

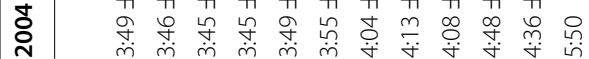

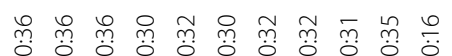
H H H H H H H H H H H

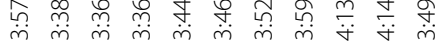

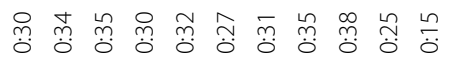
H H H H H H H H H H H

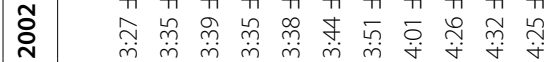
娄 H H H H H H H H H H H

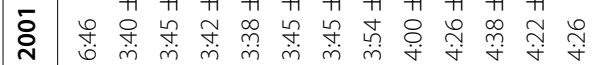
‡. H H H H H H H H H H H 品 $\quad \begin{aligned} & + \\ & \dot{m}\end{aligned}$

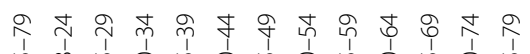

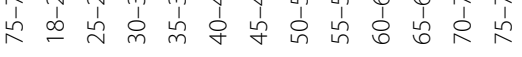
$\stackrel{\frac{c}{N}}{\Sigma}$ 
Table 4 Results of the mixed effects regression analyses for race time in the 'Jungfrau Marathon'

\begin{tabular}{|c|c|c|c|c|c|}
\hline Parameter & Estimate & Standard error & $d f$ & $t$ & $p$ value \\
\hline \multicolumn{6}{|l|}{$18-24$} \\
\hline Constant term & -1468.67 & 656.37 & 984.51 & -2.23 & 0.025 \\
\hline Female sex & 20.32 & 3.67 & 755.22 & 5.53 & $<0.0001$ \\
\hline Calendar year & 0.88 & 0.32 & 984.55 & 2.70 & 0.007 \\
\hline \multicolumn{6}{|l|}{$25-29$} \\
\hline Constant term & -1627.79 & 401.69 & 2542.02 & -4.05 & $<0.0001$ \\
\hline Female sex & 18.26 & 2.17 & 2073.43 & 8.38 & $<0.0001$ \\
\hline Calendar year & 0.95 & 0.20 & 2542.07 & 4.79 & $<0.0001$ \\
\hline \multicolumn{6}{|l|}{$30-34$} \\
\hline Constant term & -1764.41 & 270.53 & 5350.93 & -6.52 & $<0.0001$ \\
\hline Female sex & 20.86 & 1.57 & 4117.81 & 13.21 & $<0.0001$ \\
\hline Calendar year & 1.02 & 0.13 & 5350.98 & 7.61 & $<0.0001$ \\
\hline \multicolumn{6}{|l|}{$35-39$} \\
\hline Constant term & -1880.69 & 222.38 & 8062.32 & -8.45 & $<0.0001$ \\
\hline Female sex & 19.38 & 1.35 & 5718.30 & 14.33 & $<0.0001$ \\
\hline Calendar year & 1.08 & 0.11 & 8062.38 & 9.79 & $<0.0001$ \\
\hline \multicolumn{6}{|l|}{$40-44$} \\
\hline Constant term & -2350.94 & 183.75 & $11,098.30$ & -12.79 & $<0.0001$ \\
\hline Female sex & 20.53 & 1.06 & 7616.11 & 19.20 & $<0.0001$ \\
\hline Calendar year & 1.32 & 0.09 & $11,098.40$ & 14.45 & $<0.0001$ \\
\hline \multicolumn{6}{|l|}{$45-49$} \\
\hline Constant term & -2008.87 & 181.75 & $10,676.10$ & -11.05 & $<0.0001$ \\
\hline Female sex & 23.67 & 1.07 & 7201.81 & 21.96 & $<0.0001$ \\
\hline Calendar year & 1.15 & 0.09 & $10,676.17$ & 12.76 & $<0.0001$ \\
\hline \multicolumn{6}{|l|}{ 54-54 } \\
\hline Constant term & -2461.63 & 202.63 & 8035.83 & -12.14 & $<0.0001$ \\
\hline Female sex & 22.71 & 1.33 & 5336.54 & 16.96 & $<0.0001$ \\
\hline Calendar year & 1.38 & 0.10 & 8035.96 & 13.72 & $<0.0001$ \\
\hline \multicolumn{6}{|l|}{$55-59$} \\
\hline Constant term & -3145.87 & 273.07 & 4263.96 & -11.52 & $<0.0001$ \\
\hline Female sex & 21.21 & 1.85 & 2894.57 & 11.46 & $<0.0001$ \\
\hline Calendar year & 1.73 & 0.13 & 4264.08 & 12.72 & $<0.0001$ \\
\hline \multicolumn{6}{|l|}{$60-64$} \\
\hline Constant term & -3685.21 & 374.10 & 2154.69 & -9.85 & $<0.0001$ \\
\hline Female sex & 17.95 & 2.92 & 1363.42 & 6.14 & $<0.0001$ \\
\hline Calendar year & 2.00 & 0.18 & 2154.72 & 10.75 & $<0.0001$ \\
\hline \multicolumn{6}{|l|}{$65-69$} \\
\hline Constant term & -3880.01 & 616.55 & 794.88 & -6.29 & $<0.0001$ \\
\hline Female sex & 18.38 & 5.31 & 493.15 & 3.46 & 0.001 \\
\hline Calendar year & 2.10 & 0.30 & 794.89 & 6.85 & $<0.0001$ \\
\hline \multicolumn{6}{|l|}{$70-74$} \\
\hline Constant term & -2739.43 & 1248.46 & 156.09 & -2.19 & 0.030 \\
\hline Female sex & 6.60 & 8.59 & 100.46 & 0.76 & 0.444 \\
\hline Calendar year & 1.54 & 0.62 & 156.09 & 2.48 & 0.014 \\
\hline \multicolumn{6}{|l|}{$75-79$} \\
\hline Constant term & 3023.71 & 4920.13 & 11.05 & 0.61 & 0.551 \\
\hline Calendar year & -1.32 & 2.44 & 11.06 & -0.54 & 0.598 \\
\hline
\end{tabular}


Table 5 Results of the mixed effects regression analyses for race time in the 'Lausanne Marathon'

\begin{tabular}{|c|c|c|c|c|c|}
\hline Parameter & Estimate & Standard error & $d f$ & $t$ & $\mathrm{p}$ value \\
\hline \multicolumn{6}{|l|}{$18-24$} \\
\hline Constant term & -1671.42 & 652.21 & 775.44 & -2.56 & 0.011 \\
\hline Female sex & 25.36 & 3.65 & 729.37 & 6.94 & $<0.0001$ \\
\hline Calendar year & 0.94 & 0.32 & 775.44 & 2.91 & 0.004 \\
\hline \multicolumn{6}{|l|}{$25-29$} \\
\hline Constant term & -54.73 & 399.06 & 1839.49 & -0.13 & 0.891 \\
\hline Female sex & 26.47 & 2.23 & 1660.61 & 11.86 & $<0.0001$ \\
\hline Calendar year & 0.13 & 0.19 & 1839.50 & 0.70 & 0.483 \\
\hline \multicolumn{6}{|l|}{$30-34$} \\
\hline Constant term & -556.59 & 305.88 & 2861.85 & -1.82 & 0.069 \\
\hline Female sex & 26.87 & 1.79 & 2595.44 & 15.00 & $<0.0001$ \\
\hline Calendar year & 0.38 & 0.15 & 2861.88 & 2.54 & 0.011 \\
\hline \multicolumn{6}{|l|}{$35-39$} \\
\hline Constant term & -1098.15 & 247.61 & 3963.50 & -4.43 & $<0.0001$ \\
\hline Female sex & 23.05 & 1.48 & 3494.68 & 15.49 & $<0.0001$ \\
\hline Calendar year & 0.65 & 0.12 & 3963.51 & 5.33 & $<0.0001$ \\
\hline \multicolumn{6}{|l|}{$40-44$} \\
\hline Constant term & -703.52 & 241.15 & 4445.91 & -2.91 & 0.004 \\
\hline Female sex & 24.70 & 1.40 & 3881.69 & 17.53 & $<0.0001$ \\
\hline Calendar year & 0.46 & 0.12 & 4445.94 & 3.85 & $<0.0001$ \\
\hline \multicolumn{6}{|l|}{$45-49$} \\
\hline Constant term & -546.72 & 278.20 & 3485.36 & -1.96 & 0.049 \\
\hline Female sex & 29.93 & 1.61 & 2969.98 & 18.53 & $<0.0001$ \\
\hline Calendar year & 0.38 & 0.13 & 3485.35 & 2.79 & 0.005 \\
\hline \multicolumn{6}{|l|}{$50-54$} \\
\hline Constant term & -771.09 & 333.87 & 2366.70 & -2.31 & 0.021 \\
\hline Female sex & 28.46 & 2.08 & 2005.98 & 13.67 & $<0.0001$ \\
\hline Calendar year & 0.50 & 0.16 & 2366.72 & 3.02 & 0.003 \\
\hline \multicolumn{6}{|l|}{$55-59$} \\
\hline Constant term & -1001.66 & 493.28 & 1277.73 & -2.03 & 0.043 \\
\hline Female sex & 31.87 & 3.07 & 1026.03 & 10.36 & $<0.0001$ \\
\hline Calendar year & 0.62 & 0.24 & 1277.73 & 2.52 & 0.012 \\
\hline \multicolumn{6}{|l|}{$60-64$} \\
\hline Constant term & -1639.41 & 705.31 & 643.37 & -2.32 & 0.020 \\
\hline Female sex & 44.02 & 4.73 & 542.93 & 9.28 & $<0.0001$ \\
\hline Calendar year & 0.94 & 0.35 & 643.37 & 2.68 & 0.007 \\
\hline \multicolumn{6}{|l|}{$65-69$} \\
\hline Constant term & -2890.45 & 1215.31 & 217.99 & -2.37 & 0.018 \\
\hline Female sex & 43.60 & 8.10 & 178.72 & 5.38 & $<0.0001$ \\
\hline Calendar year & 1.57 & 0.60 & 217.99 & 2.60 & 0.010 \\
\hline \multicolumn{6}{|l|}{$70-74$} \\
\hline Constant term & -2691.53 & 2308.33 & 56.65 & -1.16 & 0.248 \\
\hline Female sex & 89.32 & 21.71 & 53.54 & 4.11 & $<0.0001$ \\
\hline Calendar year & 1.47 & 1.14 & 56.65 & 1.28 & 0.203 \\
\hline \multicolumn{6}{|l|}{$75-79$} \\
\hline Constant term & -669.16 & 4482.108462 & 13.40 & -0.14 & 0.884 \\
\hline Female sex & 43.69 & 26.92 & 9.86 & 1.62 & 0.136 \\
\hline Calendar year & 0.49 & 2.23 & 13.40 & 0.22 & 0.829 \\
\hline
\end{tabular}


Table 6 Sex difference (\%) in age groups in the mountain (upper two panels) and the city marathon (lower two panels)

\begin{tabular}{|c|c|c|c|c|c|c|c|c|c|c|c|c|c|c|c|c|c|}
\hline Age group & 2000 & 2001 & 2002 & 2003 & 2004 & 2005 & 2006 & 2007 & 2008 & 2009 & 2010 & 2011 & 2012 & 2013 & 2014 & $r^{2}$ & $p$ \\
\hline \multicolumn{18}{|c|}{ Jungfrau Marathon } \\
\hline $18-24$ & 8 & 6 & 8 & 8 & 11 & 8 & 5 & 4 & 3 & 5 & 10 & 13 & 7 & 4 & 10 & 0.001 & 0.906 \\
\hline $25-29$ & 4 & 5 & 3 & 5 & 3 & 4 & 6 & 3 & 5 & 4 & 6 & 8 & 5 & 7 & 10 & 0.463 & 0.005 \\
\hline $30-34$ & 9 & 8 & 6 & 5 & 5 & 3 & 4 & 7 & 4 & 7 & 4 & 7 & 9 & 8 & 9 & 0.047 & 0.436 \\
\hline $35-39$ & 7 & 8 & 6 & 7 & 6 & 8 & 6 & 5 & 6 & 6 & 7 & 5 & 3 & 7 & 6 & 0.218 & 0.079 \\
\hline $40-44$ & 12 & 10 & 4 & 6 & 6 & 8 & 7 & 6 & 7 & 5 & 7 & 5 & 5 & 6 & 6 & 0.278 & 0.043 \\
\hline $45-49$ & 9 & 9 & 6 & 8 & 8 & 9 & 8 & 7 & 5 & 8 & 6 & 6 & 8 & 7 & 9 & 0.066 & 0.354 \\
\hline $50-54$ & 11 & 12 & 10 & 7 & 5 & 6 & 5 & 8 & 5 & 9 & 7 & 7 & 6 & 8 & 7 & 0.195 & 0.098 \\
\hline $55-59$ & 5 & 8 & 11 & 4 & 5 & 8 & 6 & 7 & 9 & 8 & 4 & 4 & 7 & 3 & 7 & 0.066 & 0.353 \\
\hline $60-64$ & 3 & 2 & 3 & 1 & 2 & 3 & 4 & 5 & 5 & 8 & 7 & 6 & 8 & 8 & 8 & 0.820 & $<0.0001$ \\
\hline $65-69$ & 7 & 6 & & 7 & 4 & 2 & 3 & 3 & 5 & 2 & 1 & 11 & 8 & 6 & 9 & 0.046 & 0.457 \\
\hline $70-74$ & 1 & 14 & & & & & & & & & 4 & 7 & & 3 & 7 & 0.024 & 0.685 \\
\hline \multicolumn{18}{|c|}{ Lausanne Marathon } \\
\hline $18-24$ & 12 & 29 & 6 & 1 & 1 & 21 & 10 & 5 & 10 & 12 & 6 & 7 & 1 & 21 & 14 & 0.005 & 0.792 \\
\hline $25-29$ & 11 & 21 & 7 & 9 & 10 & 21 & 11 & 13 & 13 & 1 & 5 & 13 & 9 & 4 & 11 & 0.141 & 0.166 \\
\hline $30-34$ & 10 & 19 & 7 & 13 & 10 & 22 & 8 & 12 & 15 & 7 & 6 & 8 & 11 & 10 & 10 & 0.099 & 0.252 \\
\hline $35-39$ & 8 & 17 & 13 & 13 & 10 & 10 & 8 & 4 & 4 & 7 & 7 & 8 & 11 & 7 & 10 & 0.192 & 0.102 \\
\hline $40-44$ & 9 & 15 & 11 & 8 & 10 & 14 & 14 & 11 & 9 & 11 & 7 & 8 & 11 & 11 & 5 & 0.176 & 0.119 \\
\hline $45-49$ & 13 & 28 & 14 & 16 & 6 & 18 & 9 & 10 & 7 & 7 & 15 & 8 & 11 & 12 & 8 & 0.241 & 0.063 \\
\hline $50-54$ & 14 & 21 & 6 & 10 & 8 & 23 & 12 & 14 & 6 & 16 & 7 & 6 & 6 & 7 & 5 & 0.265 & 0.049 \\
\hline $55-59$ & 17 & 15 & 4 & 10 & 6 & 30 & 8 & 22 & 15 & 6 & 9 & 14 & 4 & 8 & 8 & 0.071 & 0.335 \\
\hline $60-64$ & 13 & 19 & & 26 & 19 & & 1 & 19 & 8 & 25 & 23 & 8 & 11 & 10 & 26 & 0.002 & 0.865 \\
\hline $65-69$ & 19 & 2 & 14 & 10 & 11 & & & 29 & 29 & 31 & 1 & & 22 & 15 & 14 & 0.051 & 0.480 \\
\hline $70-74$ & & & & & 26 & & & 20 & & & & & 39 & & & 0.590 & 0.442 \\
\hline $75-79$ & & 53 & & & & & & & & & & & & 2 & & & \\
\hline
\end{tabular}

'Lausanne Marathon' could be a specific phenomenon for Swiss endurance races. A recent study investigating all half and full marathons in Switzerland held from 2000 to 2010 showed that that participation increased in half marathons but decreased in full marathons (Anthony et al. 2014). This might be due to the fact that city marathons exist in almost every major European city. Only few marathons around the world are considered 'World Marathon Majors' and attract tens of thousands of athletes (http://www.worldmarathonmajors.com). The 'New York City Marathon' is one of them (Jokl et al. 2004, Lepers and Cattagni 2012). Most other city marathons attract much less runner. Considering performance, half-marathoners stabilized their race times whereas marathoners improved (Anthony et al. 2014). However, similarly to the studies of Jokl et al. (2004) and Lepers and Cattagni (2012), Anthony et al. (2014) considered the ten fastest of each age group.

These disparate findings for the 'Jungfrau Marathon' concerning a decrease in performance compared to the 'New York City Marathon' with an increase in performance for age group athletes (Jokl et al. 2004; Lepers and Cattagni 2012) might be explained by the different analyses or by local differences as discussed above. Future studies might investigate participation and performance trends in other mountain marathons or mountain ultra-marathons held in other countries such as Switzerland.

\section{Sex difference}

The sex difference increased in the 'Jungfrau Marathon' in age groups 25-29 and 60-64 years but decreased in 40-44 years. In the 'Lausanne Marathon', the sex difference showed no changes. Overall, the sex differences were lower in the 'Jungfrau Marathon' ( 6-7 \%) compared to the 'Lausanne Marathon' where the sex differences were $\sim 10-12 \%$ from age group $18-24$ to $55-59$ years. For age groups 60-64 to 70-74 years, the sex difference continuously increased. Similar findings have been reported by Senefeld et al. (2015) for age group marathoners, where the sex difference increased across age groups from $10.6 \pm 0.5 \%$ for the $25-29$-year-olds to $23.3 \pm 2.6 \%$ for the 80-84-year-olds. The increase in sex difference in marathon running with increasing age is most likely due to the lower number of women finishers than men (Hunter and Stevens 2013). Considering our own data, 
the men-to-women ratio increased in the mountain marathon mainly in the years 2008-2014 whereas in the city marathon, the increase was in earlier years between 2000 and 2008. Obviously, there was a shift in the mento-women ratio across calendar years between city and mountain marathon running in Switzerland.

\section{Limitations and implications for future research}

Findings from the 'Jungfrau Marathon' were compared to those of a 'non-mountainous' or 'non-hilly' city marathon. It would have been more appropriate to compare the 'Jungfrau Marathon' findings to those of a hilly marathon run mostly on paved surface. Then performance comparison would be more valid. Future studies might consider this aspect.

A further limitation is that the present findings are mainly relevant for Switzerland. Future studies might investigate other mountain marathons such as the 'Rab Mountain Marathon' (http://www.rabmountainmarathon.com) or the 'Raccoon Mountain Marathon' (http:// runchattanooga.org/rmm/).

\section{Conclusions}

In summary, performance decreased in age group athletes competing in 'Jungfrau Marathon' although participation increased. This finding for one of the most famous mountain marathons is different to findings for a large city marathon such as the 'New York City Marathon'. These disparate findings might be explained by the different data analyses or as a typical phenomenon of races held in Switzerland. Future studies might investigate participation and performance trends in other mountain marathons or mountain ultra-marathons held in other countries such as Switzerland.

\section{Authors' contributions}

All authors have been involved in collecting data, writing, drafting and revising the manuscript. BK and MZ collected all data. CR performed the statistical analyses. BK drafted the manuscript. TR participated in the design of the study and revised the manuscript critically for important intellectual content. All authors read and approved the final manuscript.

\section{Author details}

${ }^{1}$ Gesundheitszentrum St. Gallen, Vadianstrasse 26, 9001 St. Gallen, Switzerland.

${ }^{2}$ Institute of Primary Care, University of Zurich, Zurich, Switzerland.

Compliance with ethical guidelines

\section{Competing interests}

The authors declare that they have no competing interests.

\section{References}

Ahmadyar B, Rüst CA, Rosemann T, Knechtle B (2015) Participation and performance trends in elderly marathoners in four of the world's largest marathons during 2004-2011. Springerplus 4:465

Anthony D, Rüst CA, Cribari M, Rosemann T, Lepers R, Knechtle B (2014) Differences in participation and performance trends in age group half and full marathoners. Chin J Physiol 57:209-219

Aschmann A, Knechtle B, Cribari M, Rüst CA, Onywera V, Rosemann T, Lepers R (2013) Performance and age of African and non-African runners in halfand full marathons held in Switzerland, 2000-2010. Open Access J Sports Med 4:183-192

Cribari M, Rüst CA, Rosemann T, Onywera V, Lepers R, Knechtle B (2013) Participation and performance trends of East-African runners in Swiss halfmarathons and marathons held between 2000 and 2010. BMC Sports Sci Med Rehabil 5:24

Gloor RU, Knechtle B, Knechtle P, Rüst CA, Haupt S, Rosemann T, Lepers R (2013) Sex-related trends in participation and performance in the'Swiss Bike Masters'from 1994-2012. Percept Mot Skills 116:640-654

Haupt S, Knechtle B, Knechtle P, Rüst CA, Rosemann T, Lepers R (2013) The age-related performance decline in ultraendurance mountain biking. Res Sports Med 21:146-158

Hunter SK, Stevens AA (2013) Sex differences in marathon running with advanced age: physiology or participation? Med Sci Sports Exerc 45:148-156

Jokl P, Sethi PM, Cooper AJ (2004) Master's performance in the New York City Marathon 1983-1999. Br J Sports Med 38:408-412

Lepers R, Cattagni T (2012) Do older athletes reach limits in their performance during marathon running? Age (Dordr) 34:773-781

Leyk D, Erley O, Ridder D, Leurs M, Rüther T, Wunderlich M, Sievert A, Baum K, Essfeld D (2007) Age-related changes in marathon and half-marathon performances. Int J Sports Med 28:513-517

Leyk D, Erley O, Gorges W, Ridder D, Rüther T, Wunderlich M, Sievert A, Essfeld D, Piekarski C, Erren T (2009) Performance, training and lifestyle parameters of marathon runners aged 20-80 years: results of the PACE-study. Int J Sports Med 30:360-365

Leyk D, Rüther T, Wunderlich M, Sievert A, Essfeld D, Witzki A, Erley O, Küchmeister G, Piekarski C, Löllgen H (2010) Physical performance in middle age and old age: good news for our sedentary and aging society. Dtsch Arztebl Int 107:809-816

Reaburn P, Dascombe B (2008) Endurance performance in masters athletes. Eur Rev Aging Phys Act 5:31-42

Rüst CA, Knechtle B, Knechtle P, Rosemann T, Lepers R (2012) Age of peak performance in elite male and female Ironman triathletes competing in Ironman Switzerland, a qualifier for the Ironman world championship, Ironman Hawaii, from 1995 to 2011. Open Access J Sports Med 3:175-182

Rüst CA, Knechtle B, Knechtle P, Pfeifer S, Rosemann T, Lepers R, Senn O (2013) Gender difference and age-related changes in performance at the longdistance duathlon. J Strength Cond Res 27:293-301

Senefeld J, Joyner MJ, Stevens A, Hunter SK (2015) Sex differences in elite swimming with advanced age are less than marathon running. Scand J Med Sci Sports. doi:10.1111/sms.12412 [Epub ahead of print]

Teutsch U, Knechtle B, Rüst CA, Rosemann T, Lepers R (2013) Age group athletes in inline skating: decrease in overall and increase in master athlete participation in the longest inline skating race in Europe- - the Inline One-Eleven. Int J Gen Med 6:345-355

Van der Worp MP, ten Haaf DS, van Cingel R, de Wijer A, Nijhuis-van der Sanden MW, Staal JB (2015) Injuries in runners; a systematic review on risk factors and sex differences. PLoS One 10(2):e0114937

Zingg MA, Knechtle B, Rüst CA, Rosemann T, Lepers R (2013) Reduced performance difference between sexes in master mountain and city marathon running. Int J Gen Med 6:267-275

Received: 17 August 2015 Accepted: 9 September 2015

Published online: 18 September 2015 